\title{
JWST Cryo Fine Guidance Closed Loop Test Results
}

\author{
M. Begoña Vila* ${ }^{\text {b,a }}$, Scott D. Lambros ${ }^{\mathrm{c}, \mathrm{a}}$, David M. Diaz ${ }^{\mathrm{d}}$, Henry Fu ${ }^{\mathrm{d}}$, Steven S. Lee ${ }^{\mathrm{d}}$, Luis Meza , \\ Kevin J. Phillips ${ }^{\text {ea }}$, Ben del Rosario ${ }^{\text {, }}$ J. Carl Wu ${ }^{\text {f,a }}$ \\ a \\ b \\ NASA’s Goddard Space Flight Center, 8800 Greenbelt Rd, Greenbelt, MD 20771; Stinger Ghaffarian \\ Technologies, 7701 Greenbelt Rd \#400, Greenbelt, MD 20770; ATA Aerospace, 7474 Greenway Center Dr., \\ d \\ Greenbelt, Md. 20770; Northrop Grumman Aerospace Systems, One Space Park, Redondo Beach, CA \\ 90278; Microtel LLC, 7703 Belle Point Dr, Greenbelt, MD 20770; the Hammers Company, 7500 Greenway \\ Center Dr \#1500, Greenbelt, MD 20770
}

\begin{abstract}
Keywords: Fine Guiding, Closed Loop, Attitude Control, Pointing, Stability, Control, James Webb Space Telescope, Line of Sight

The James Webb Space Telescope uses the Fine Guidance Controller to achieve pointing accuracy to a millionth of a degree needed for its scientific observations. This closed loop controller includes the Fine Guidance Sensor instrument, the Attitude Control System, and the Fine Steering Mirror, all working together to generate precise attitude updates every $64 \mathrm{~ms}$ to stabilize and point the Observatory. It was exercised for the first time with the flight hardware during the cryogenic test at Johnson Space Center. We provide a top level summary of the test, the results, and its performance in preparation for on-orbit operations.
\end{abstract}

\section{INTRODUCTION}

The James Webb Space Telescope (JWST) will be the next premier space infrared telescope looking back in time to the first galaxies and stars that were formed 13.5 billion years ago, study star and galaxy formation, and identify exoplanets and their constituent components. The Observatory consists of a $6.5 \mathrm{~m}$ diameter, segmented, deployable primary mirror, an Integrated Science Instrument Module (ISIM), a deployable tennis-court sized sunshield, and a spacecraft element for communications, control and other housekeeping functions.

The primary mirror is made up of 18 individual hexagonal mirrors segments in order to fold up to fit in the launch vehicle, which are aligned to appear as one primary mirror, with diffraction limited performance at $2 \mathrm{um}^{1}{ }^{1}$ The ISIM consists of 4 scientific instruments and a guider, and various structural, thermal, electronic, and operational systems that support them. The instruments are a Near Infrared Camera (NIRCam) ${ }^{2,3}$, a Near Infrared Spectrograph (NIRSpec) ${ }^{4,5}$, a Mid-Infrared Instrument (MIRI) ${ }^{6,7}$, and a Fine Guidance Sensor/Near Infrared Imager and Slitless Spectrograph (FGS/NIRISS) ${ }^{8,9}$. Together they encompass the wavelength range of $0.5-28$ um giving an unprecedented view of the universe at those wavelengths. The instruments are a contribution from partners in Europe, Canada and the US.

The spacecraft attitude control system uses gyros and star trackers as sensors and reaction wheels as control actuators. This equipment will meet the coarse observatory pointing requirements of 7 arcseconds pointing accuracy and pointing stability of 1 arcsecond over each 0.1 second period. The less stringent ISIM field of view roll performance will be provided solely by the spacecraft Attitude Control System (ACS). However for scientific observations a higher milliarcsec pointing accuracy is required that is achieved by the Fine Guidance Controller which includes the Fine Guidance Sensor instrument (FGS), the ACS, and the Fine Steering Mirror (FSM).

The Fine Guidance Sensor contains 2 separate detector channels (for redundancy and greater accuracy) to provide, as the name implies, pointing error signals on selected guide stars to the Attitude Control System (ACS) for fine pointing of the Observatory. FGS provides source positions of the guide stars along with quality indicators (centroids) every 64 ms, to 
an accuracy of 4 milli-arcsecs. The objective of the test described herein is to demonstrate successful Observatory pointing by closing the loop of the FGS centroids to the ACS, and corresponding control of the Fine Steering Mirror (FSM) which adjusts pointing within $16 \mathrm{~ms}$ based on the provided centroid positions. The commanding of the FSM is done through the Actuator Drive Unit (ADU) that also commands the actuators on the other JWST mirrors. In this test the ADU is an engineering model. Each of these subsystems had been successfully tested separately. This test was the first time all subsystems were tested together with flight hardware.

The test was a cryogenic vacuum test in the chamber at the Johnson Space Center (JSC). It included the Optical Telescope Element (OTE), which is essentially the primary and secondary mirrors, an aft optics system, the fine steering mirror, and the ISIM. The integrated OTE and ISIM is referred to as OTIS. Figure 1 shows the OTIS as it exited the test chamber having just completed the cryo-vac test at JSC. The Spacecraft portion is a high fidelity simulator, with the flight ACS software. An Aft optics subsystem Source Plate Assembly (ASPA) source provides the "guide star" (GS) imaged by the FGS Guiders. Operationally, the Actuator Drive Unit (ADU) commands rotation of the Fine Steering Mirror (FSM) such that the ASPA GS image, projected on the FGS focal plane, is moved. This simulates the motion of the guide star that the Guider detectors will see on orbit as a result of Observatory drift.

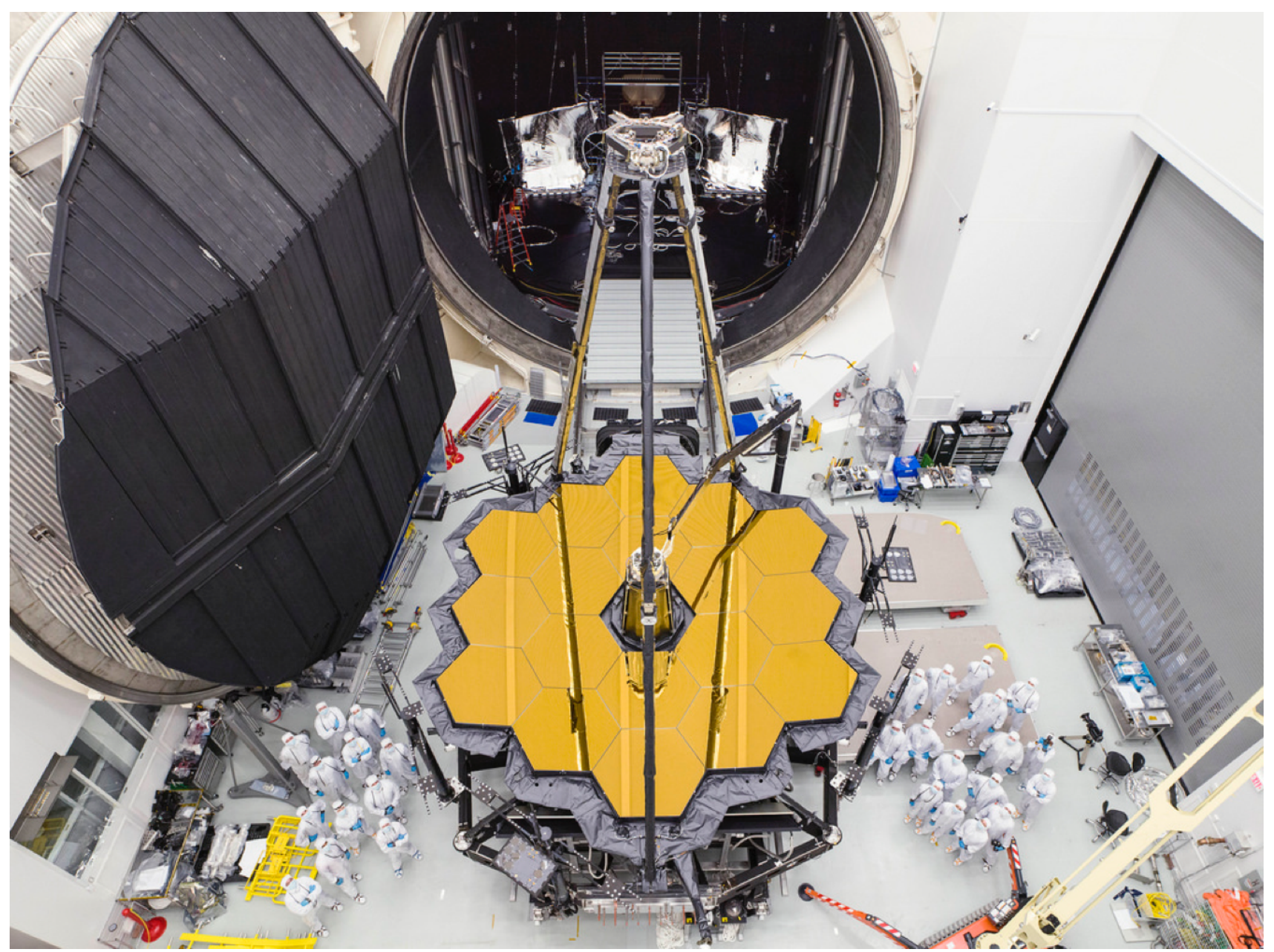

Figure 1. OTIS exiting the JSC cryo vacuum chamber (credit NASA C. Gunn) 
There are 3 distinct subsections to the test procedure, each with their own objectives: the Polarity and Alignment Test, the Functional Test, and the Timing Performance Test. Each of these is discussed below, and have their own subsections in the step-by-step procedure. The polarity between the FGS coordinates and the FSM axis was confirmed and the FGS tracking performance on a moving source validated. The ability to maintain closed loop and FGS lock while tracking and fine guiding with the Guide Star moving in various patterns was demonstrated as well as the timing budget. A typical observation of 10000 seconds while in fine guide closed loop and another JWST instrument taking images in parallel as it would on orbit, duplicating the data and telemetry rates that would be seen, confirmed full functionality. The successful testing identified some items to be optimized and set the fine guidance control on a good path for operations. Top level test results are included.

\section{CLOSED LOOP FUNCTIONALITY AND TEST SET UP}

\subsection{Fine Guidance Control Functionality}

The FGS guider provides the capability to identify, acquire, track, and fine guide on a Guide Star returning an error signal to the spacecraft ACS. After the Observatory has slewed to the part of the sky at the observation attitude, the guider reads the detector in stripes to identify the guide star by applying a recognition algorithm that compares the observed positions of the objects on the detector with the positions provided by the commanded catalog that contain a guide star and up to ten reference stars. After a successful identification, an acquisition of the guide star takes place using a smaller $128 \times 128$ pixel window centered on the position provided by the identification and this is followed by an acquisition in a $32 \times 32$ pixel window. For each of these activities a centroid position of the guide star is given that includes the guide star position in $\mathrm{x}$ and $\mathrm{y}$, its intensity in counts/sec on a central $3 \times 3$ pixel window and quality indicators for this centroid that include the point spread function (PSF) width, height, signal to noise, etc. A quality indicator is given to this centroid that marks it as bad if the criteria are not met.

After a successful acquisition the centroid position of the guide star is used to slew the observatory to the science attitude required by the prime science instrument. After the slew is completed another acquisition is run again with a $128 \times 128$ pixel window followed by a $32 \times 32$ window to find the guide star accurately once again. At this time a very small 'zeroangle' maneuver slew occurs to correct any remaining pointing error on the position of the guide star based on the centroid provided.

The FGS guider enters now into its guiding mode - first tracking the guide star in a 32x32 pixel window and providing a centroid every $64 \mathrm{~ms}$ to the ACS with the accurate position of the guide star in $\mathrm{x}, \mathrm{y}$, its intensity and quality indicators. With the receipt of the first valid centroid the Fine Guidance loop is engaged in closed loop where the Guider provided centroids will be reacted upon by the ACS moving the FSM, which in turn will be reflected in a position change on the guide star centroid provided by the guider. During this tracking mode, the FGS guider keeps track of each centroid and determines if the $32 \times 32$ pixel detector window needs to be moved to keep the guide star in the center of the tracking window. These are configurable parameters but the default will move the detector track window if 16 centroids in a row indicate that the guide star has moved more than 2 pixels from the center of the track window. The ACS in parallel is using the centroids provided by the guider 16 times every second to determine if a command to move the Fine Steering Mirror is needed and if so by how much. Eventually the ACS movement of the FSM will be such that the guider does not need to move the track window anymore as any movement is compensated by the ACS command to the FSM. At this point in normal operations the guider will be commanded to its fine guidance mode where an $8 \times 8$ detector window is read that provides the higher signal to noise needed for accurate pointing for science observations. When the guider is in this fine guide mode it does not move the detector window anymore but relies solely on the ACS closed loop commanding of the FSM based on the guide star centroids provided every $64 \mathrm{~ms}$ to keep the guide star in the center of that $8 \times 8$ fine guidance window.

There are special operations on orbit where the Fine Guidance system remains in track mode - one is for moving target operations for science observations of objects in the solar system where the target moves across the guider field of view in an established pattern and the guider moves the tracking window accordingly. The other is in the early stages of the Observatory commissioning where the mirrors are not yet aligned and the image of the guide stars are aberrated and a $32 \times 32$ detector window size is needed to capture it. In this paper we will not talk about the moving target operations but some of the parameter updates which were used in this test are applicable to the commissioning activities. 
The Fine Guidance loop is determined by the Spacecraft's minor time code of $64 \mathrm{~ms}$ that determines when the integration on the guider detector will start and when the centroids that are being generated will be picked up by the ACS. The operation of the guider has been extensively verified both by analysis, in simulators and testing with the flight hardware and simulated optical sources to confirm it does generate a centroid position every 64 ms, with a latency of 40 $\mathrm{ms}$ - latency being defined as the time between the middle of the integration in the detector and the time when the centroid is picked up on the next $64 \mathrm{~ms}$ Spacecraft minor cycle. ${ }^{10,11,12,13}$ The performance of the ACS has been verified by analysis and on simulators and the operation of the FSM testing at lower level. ${ }^{14}$ REF NGAS please add information if any you would like to provide about the ACS and FSM operation.

The first and only time when the closed loop could be tested until being on orbit was in the OTIS cryo testing at JSC where the guider flight hardware and the Fine Steering Mirror hardware were present with the ACS algorithm present in the Spacecraft simulator software. An optical source was available to simulate a star and the tracking and fine guidance closed loop performance was validated.

\subsection{OTIS Sources and Test Set Up}

An optical source was used during the OTIS test to simulate the stars to be used on orbit. Those sources were optimized to test the alignment of the JWST mirrors but were not optimal to test closed loop operations. Figure 2 shows what the optical source looked like on the guider detector, compared with how a star would look during normal on orbit operations. During the early stages of commissioning when the mirrors are not yet aligned the stars will look as the third image in Figure 2.

The guider has a set of parameters to determine the quality of the centroids based on the expected on-orbit PSFs. From Figure 2 it is clear that the OTIS source would not meet these requirements which would cause the centroids to be marked as 'bad'. The ACS will not use a centroid that is marked bad for calculating the mirror positions so the parameters that define a good centroid on the FGS flight software needed to be updated so they would pass and declare the centroid as good. Lots of analysis and testing on the simulators were run prior to the OTIS cryo test to optimize what those new parameters should be and as a result the following were relaxed: the PSF width and height, the subwindow sizes used by the software to calculate signal and noise levels and their value limits.

Note that during the early stages of commissioning when the mirrors are not yet aligned similar updates are needed for those parameters to be able to guide on a typical guide star as shown in the global alignment image in Figure 2.

\section{a) OTIS}

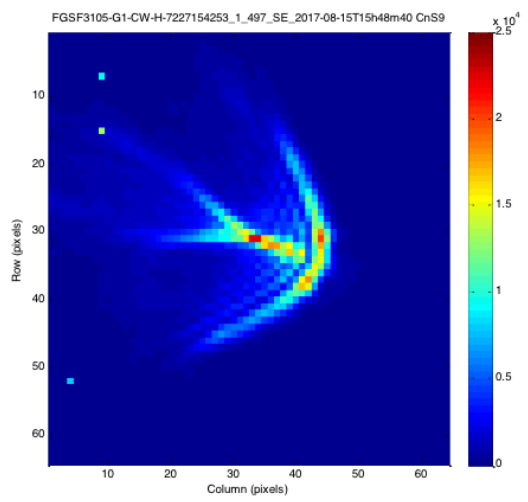

b) On orbit normal ops

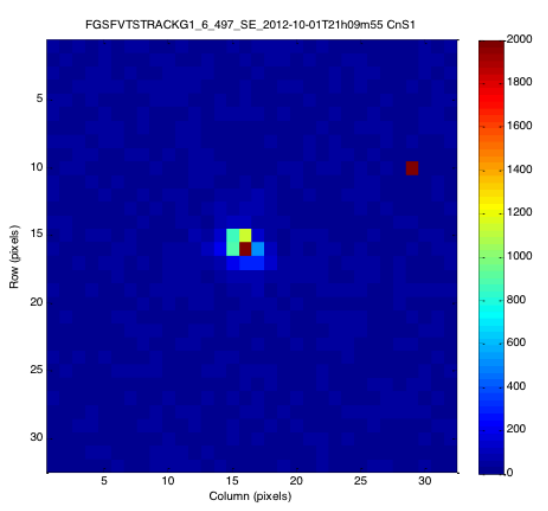

c) On orbit global alignment

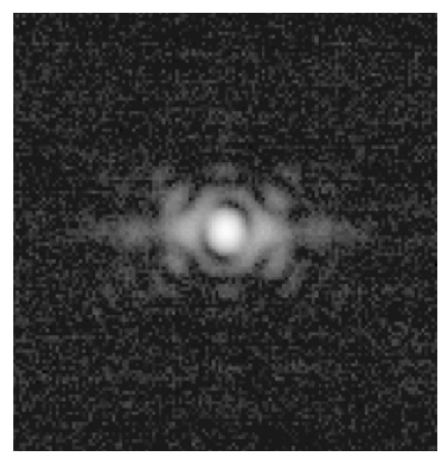

Figure 2. Source images on the FGS detector 32x32 pixels: (a) OTIS source used for closed loop testing; (b) Example of a typical on-orbit guide star image; (c) Simulation of a source as seen during early commissioning before the mirrors are aligned.

Another update needed for OTIS testing is because the simulated optical source could not be moved whereas a normal star on orbit would appear to move due to the Observatory drift. So to confirm the closed loop operation it was necessary 
to have movement on that optical source so the FSM and the guider centroids could react accordingly. The only way to achieve movement of the optical source was by commanding movements on the FSM which would move the source on the guider detector. In normal on orbit operations there is an 'ideal' position for the guide star that is defined as the 'zero' position. When the guider centroids indicate that the guide star is moving away from that zero position, the ACS reacts by moving the FSM to compensate for that movement of the guide star, and the following guider centroids would indicate that the movement was successful. In the OTIS test the FSM was used to move the optical source but when the guider centroids reported correctly that the guide star had moved there was no desire for the ACS to react moving the FSM in the opposite direction as it would do on orbit, as this would null the closed loop operations every 64 ms. Thus the 'zero' position was changed every time the FSM was moved so the reported centroid would not cause the FSM to move back.

\section{TESTS RUN AT OTIS}

This section summarizes the three tests run at OTIS and their objectives.

\subsection{Open Polarity and Alignment Check}

This test is designed to validate the polarity and alignment between movements in the FSM and the image response observed by the FGS. The polarity refers to the alignment and directionality of the FSM axes with respect to FGS sensor coordinates. Specific FSM movement patterns are used to characterize any misalignments and this information will be used to update the Spacecraft flight software alignment matrix. Because this test is run open loop, only a portion of the Fine Guidance Control (FGC) loop is exercised. Observing and post-processing the recorded FSM position commands and the ASPA GS image motion is used to validate the polarity and alignment between the FSM and FGS.

The Open Loop Polarity and Alignment Check test is broken into two complementary and sequential sections. The first, the Coarse Polarity Check, captures full frame images of the ASPA guide star before and after movements by the FSM. No centroid processing takes place during this check. The second, the Centroid Polarity and Alignment Test, uses stored command sequences to move the FSM in discrete steps that follow a defined pattern and confirm the GS location in the FGS field of view (FGS FOV) from centroids produced by FGS. This test is performed on Guider-1 and Guider-2, using both the primary and redundant ADU sides with each Guider.

Figure 3 shows the schematic representation for this test indicating the FSM being moved by the ADU and the recorded images from the guider captured on the Solid State Recorder (SSR) for analysis of the centroid position that can then be compared with the commanded position. Figure 4 shows the movement in multiple directions that were run for each guider to check polarity. Figure 5 shows the spiral pattern commanded with the guider tracking used to identify any misalignments.

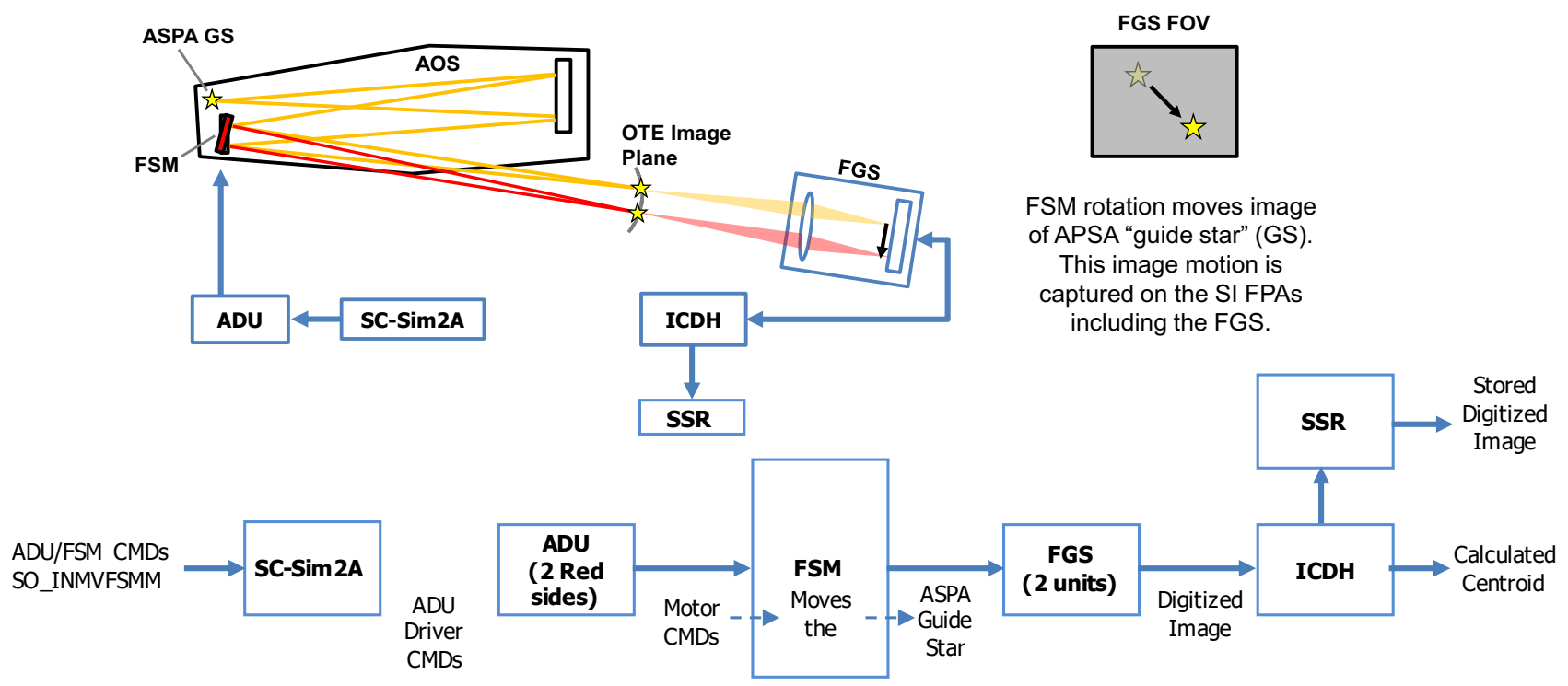

Figure 3. Schematic Representations of the Open Loop Polarity and Alignment Test Setup at JSC 
(1)
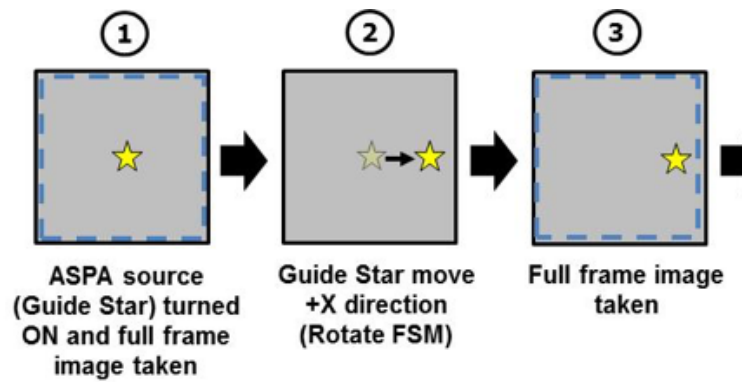

(11)

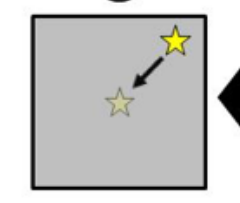
Guide Star move -X
and $-Y$ direction (Rotate FSM)

7

(13)

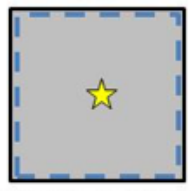

Full frame image taken

(10)
(4)
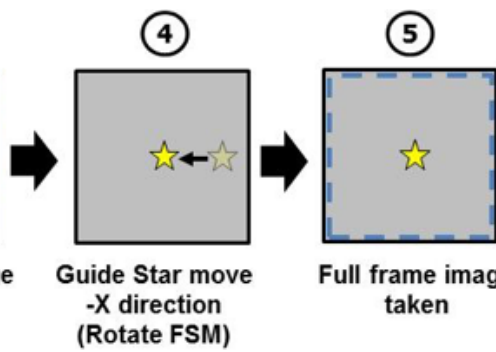

Full frame image taken

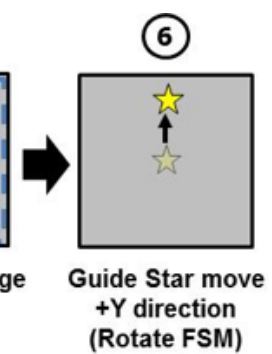

(8)

(9)
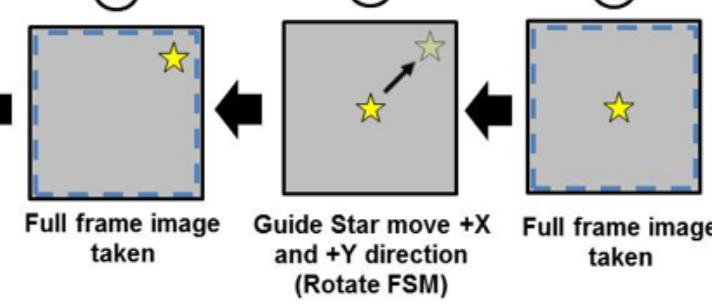

Full frame image taken

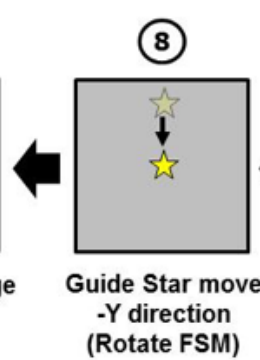

otate FSM

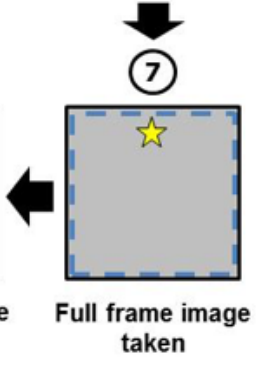

Figure 4. Illustration of the Coarse Polarity Check at OTIS

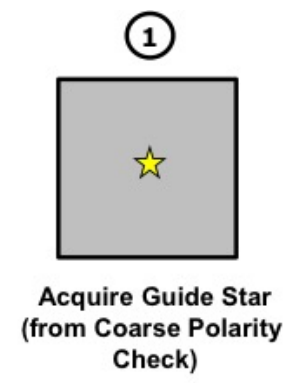

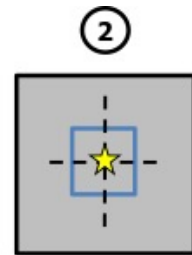

Find Centroid (FGS TRACK)

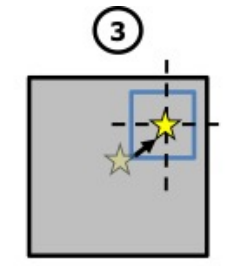

Initiate non-core SCS

Move Guide Star (Rotate FSM)

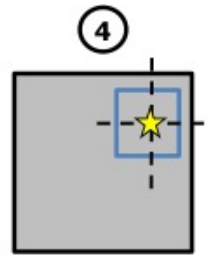

Find Centroid (FGS TRACK) And

Confirm Movement (SCS)

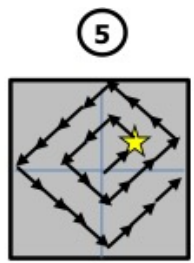

Repeat Steps 3-4 Until SCS pattern completes

Figure 5. Illustration of the Centroid Polarity and Alignment Test at OTIS

\subsection{Functional Test}

This test will validate functionality of the OTIS line of sight (LOS) Fine Guidance Control loop. The primary objective of this test is to demonstrate that Fine Guidance Control can be established and maintained with the FGS in the TRACK and FINE GUIDE modes. The test demonstrates the functionality of the OTIS LOS Fine Guidance control loop in response to Guide Star motions that are discrete steps, as well as Guide Star motions characterized by a periodic wave motion.

The Fine Guidance Control Loop Functional Test is divided into two complementary and sequential sections: the Step LOS Functional Loop test and the Dynamic LOS Functional Loop test. The first, the Step LOS Functional Loop test, 
starts with the FGS in TRACK mode while the FGS maintains lock after multiple step LOS movements. The resulting Guide Star movements are designed to demonstrate that the TRACK window moves to keep the Guide Star within the TRACK window. The test then transitions the FGS to FINE GUIDE mode while maintaining lock after two offset step LOS movements (out and back). Once the FGS is in FINE GUIDE mode, FSM movements are within the FGS Fine Guide window (8x8 pixels).

The second, the Dynamic LOS Functional Loop test, uses a series of stored command sequences to move the FSM in discrete steps that cause the Guide Star to follow a time- based periodic wave pattern within the FGS Fine Guide window (8x8 pixels). Three test cases, 1) movement in the X-direction only, 2) movement in the Y-direction only, and 3) simultaneous movement in the $\mathrm{X}$ and $\mathrm{Y}$ directions, are executed to validate the functionality of the closed loop control system in response to dynamic inputs.

Both test sections use the Fixed Target Offset command to move the FSM. The Fixed Target Offset command introduces a FSW offset (bias) to the commanded Guide Star location. The spacecraft FSW algorithm response to the FSW offset causes the movement to the FSM. This test uses Guider 1 for both sections and Guider 2 for the Step Functional only.

Figure 6 shows the schematic representation for this test indicating the closed loop operations with FSM being moved by the ADU and the recorded images and centroids from the guider being captured.
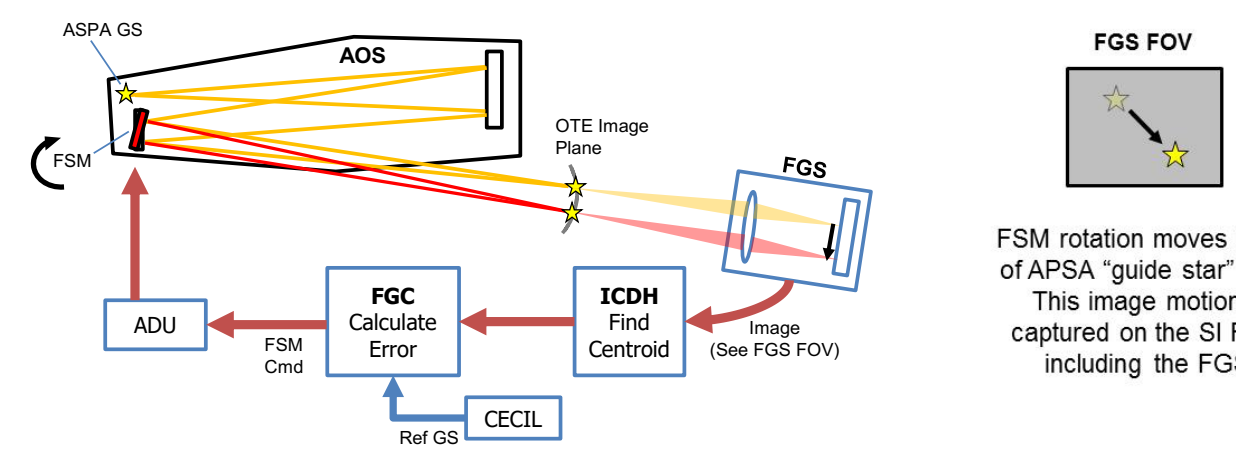

FSM rotation moves image of APSA "guide star" (GS).

This image motion is captured on the SI FPAs including the FGS

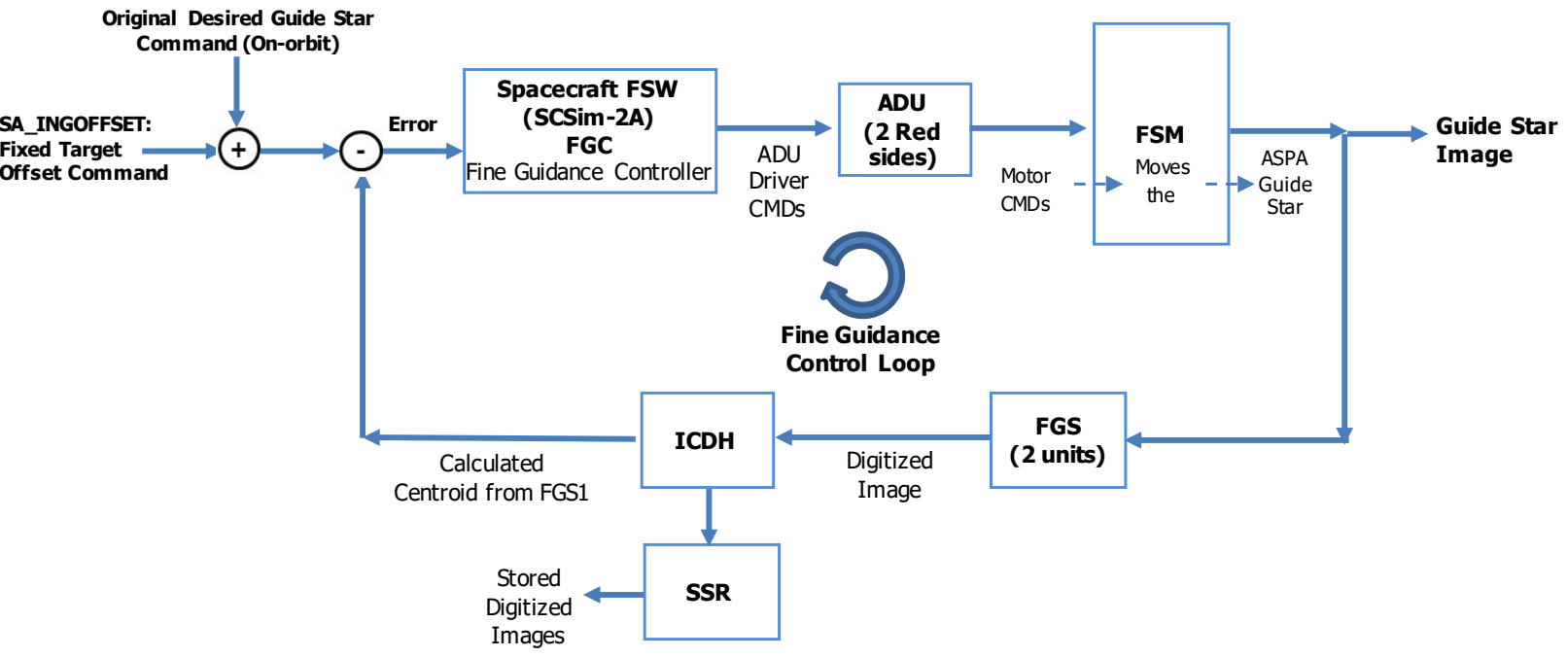

Figure 6. Schematic Representations of the Fine Guidance Control Loop Functional Test Setup

Figure 7 shows the step LOS functional closed loop test with the guider in track mode moving the $32 \times 32$ pixel detector window as the FSM moves the source position and with the guider in fine guide mode where the $8 \times 8$ pixel detector window is not moved but the source position moves within that window. 
FGS TRACK Mode

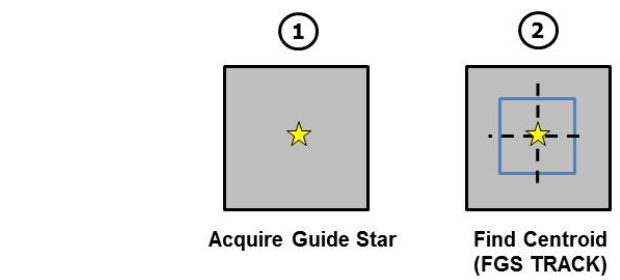

FGS FINE GUIDE Mode

(6)

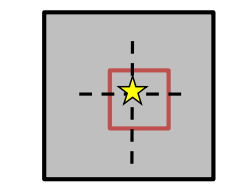

Enter FGS

FINE GUIDE Mode and

Collect Centroid Data
(2)

(FGS TRACK)
(4)

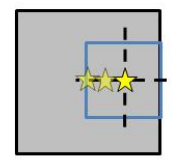

Move GS

Until FGS TRACK

Box moves and

Offset while in FGS

step

(ㄱ)

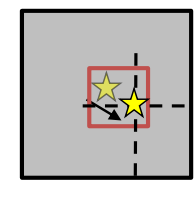

Cmd GS

Offset while in FGS

FINE GUIDE and

Collect Centroid Data
(8)

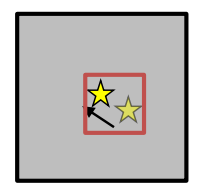

Cmd GS

back to original

position
(5)

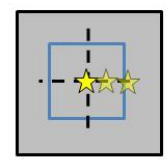

Return GS and FGS

TRACK Box back to
Original position and

Original position and
Collect Centroid Data

Figure 7. Illustration of the Step LOS Functional Loop Test

Figure 8 shows the dynamic portion of the functional test with a continuous move of the FSM in multiple directions to confirm the closed loop is maintained with the guider in Fine Guide mode.

- Case A: Line-Of-Sight (LOS) in X Motion only
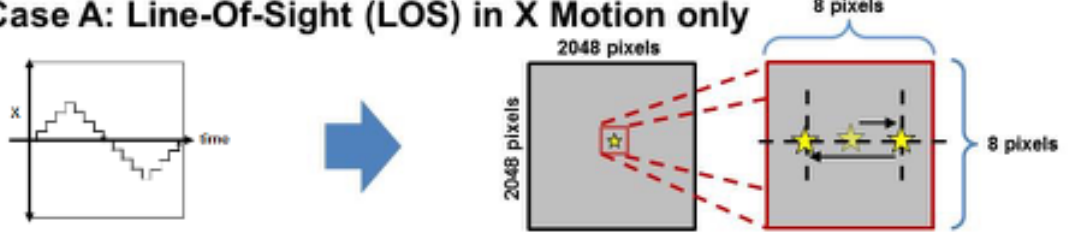

- Case B: Line-Of-Sight (LOS) in Y Motion only
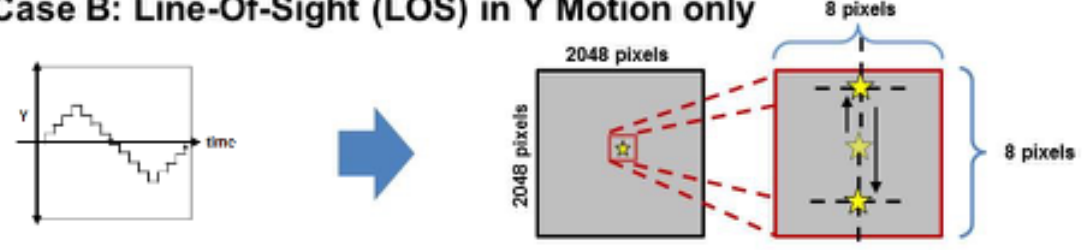

- Case C: Line-Of-Sight (LOS) in $\mathrm{X}$ and $\mathrm{Y}$ motion
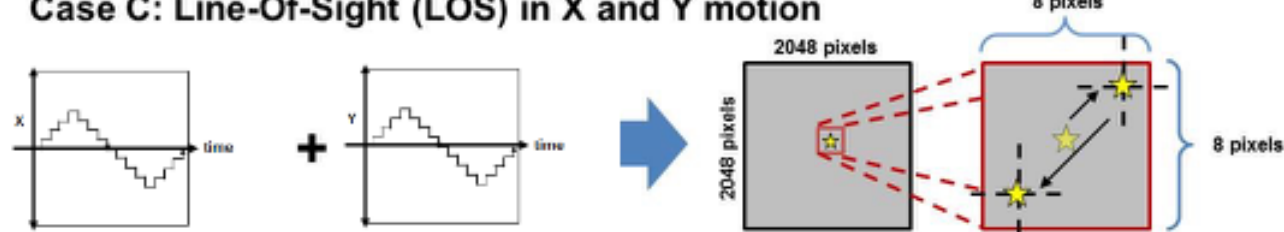

Figure 8. Illustration of the three test cases for the Time-based Dynamic LOS Functional Loop Test 


\subsection{Timing Performance Test}

This test will validate the end to end data latency of the OTIS LOS Fine Guidance Control loop. The primary objective of this test is to demonstrate that the calculated end to end loop latency is consistent with the OTIS LOS Fine Guidance Control Loop timing requirements with the FGS in FINE GUIDE mode.

The timing performance of the Fine Guidance Control Loop is validated by 1) maintaining fine guidance lock in FINE GUIDE mode during the test, and 2) using test telemetry collected during the test, measuring an end to end latency that is less than the sum of the OTIS LOS Fine Guidance Control Loop timing requirements.

The end to end loop latency is defined as the elapsed time from the middle of the FGS detector integration of the simulated guide star to when the guide star position changes due to the FSM motion in response to the FGS centroid. One Guider is used by the Control Loop to maintain fine guide control and to determine the time of the middle of the detector integration (start of latency). However as the guider generates a centroid every $64 \mathrm{~ms}$ the actual time when the FSM mirror moved the guide star cannot be captured with millisecond accuracy required to validate the end to end loop latency. Therefore the other guider is used in parallel taking fast $8 \times 8$ subarray images that can be analyzed to provide a finer resolution to when the source movement is seen when the FSM has moved (end of latency).

This test uses a series of stored command sequences to move the guide star position by using a fixed target offset command to introduce a flight software offset (bias). When the FSM moves the star's position moves simultaneously in both guiders. The commanded FSM motion is in a time-based square "step" wave pattern to validate the loop latency. This is a repeating pattern that results in a guide star position movement that spans positive and negative positions along the X-axis in both guiders. This is illustrated in Figure 9. The test schematic is similar to Figure 6 with the addition of the second guider taking images in parallel.

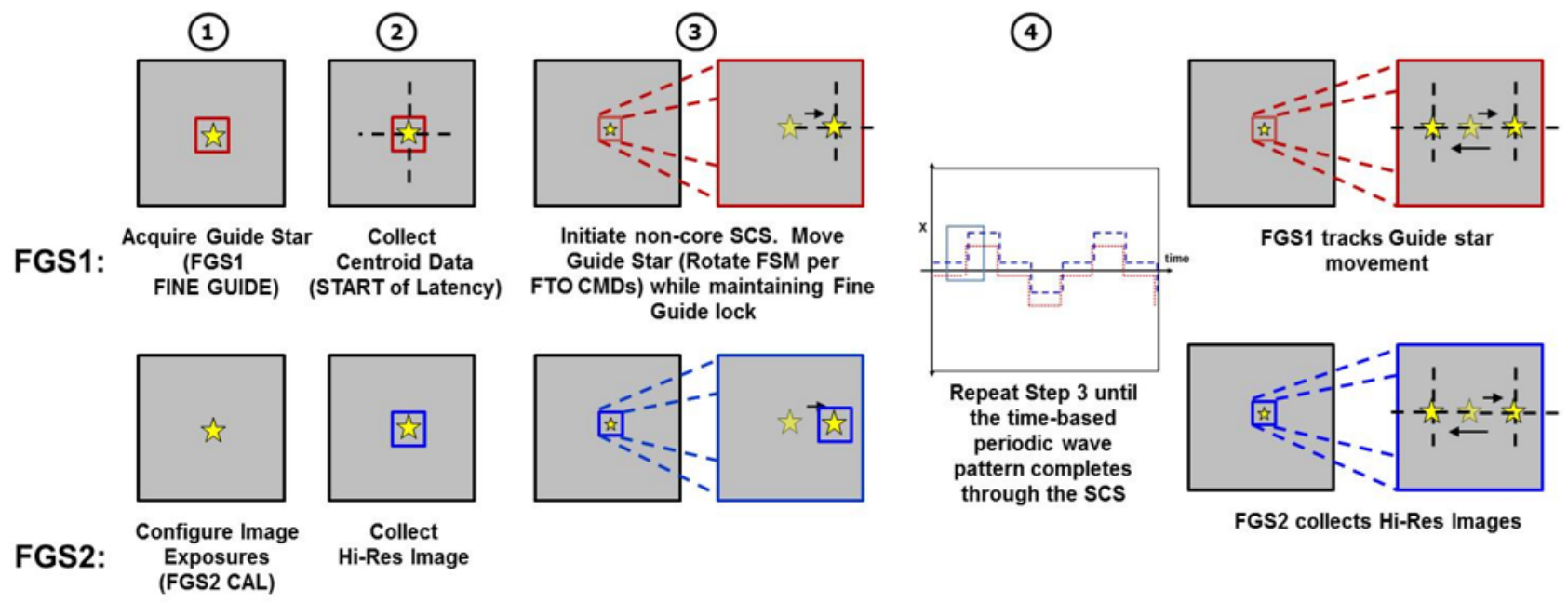

Figure 9. Illustration of the Fine Guidance Control Loop Timing Performance Test

\section{RESULTS FROM TESTS AT OTIS}

This section summarizes the results from the three tests run at the OTIS cryo-vacuum test.

\subsection{Open Polarity and Alignment Check Test Results}

The test results indicate that the polarity from the ACS/FSM to both guiders is correct - the guide star images moved in the expected direction, as shown in Figure 10. The movement seen on the guider detectors was a bit smaller than expected - instead of moving 100 pixels, the sources moved approximately 92 pixels. This $8 \%$ discrepancy will be corrected on the commanding algorithm. 
The alignment data showed good agreement of the actual centroid motion as compared to the commanded position. Each of the guiders kept the guide star within its 32x32 pixel track window box updating the track window position as needed as the FSM moved the star in the spiral pattern. A slight misalignment between the FSM commanded position and the actual centroid positions was seen for both guiders as shown in Figure 11. This slight misalignment does not impact the capability of the Fine Guidance Control loop.

\section{Fine Guidance Sensor 1 (Guider 1)}
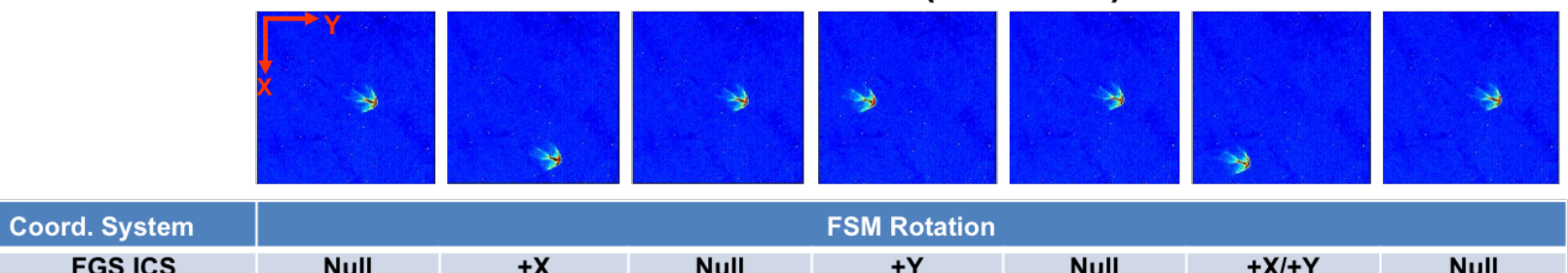

SI Pixel (in figure)

Null

FSM Rotation

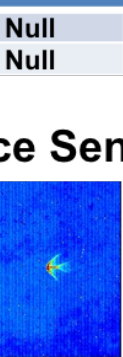

$+Y$
$-Y$

Null

$+\mathrm{X} /+\mathrm{Y}$

Null

$-Y$

Null

$+X /-Y$

Null

Fine Guidance Sensor 2 (Guider 2)
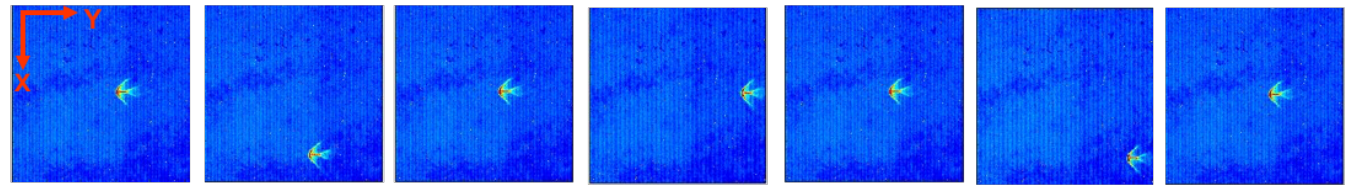

\section{Coord. System} FGS ICS

SI Pixel (in figure)

Null

$+\mathrm{X}$
$+\mathrm{X}$

\section{Null}

FSM Rotation

\begin{tabular}{l|l|l} 
Null & $+\mathbf{X}$ & Null \\
\hline
\end{tabular}

$+Y$
$+Y$

Null

\section{$+\mathrm{X} /+\mathrm{Y}$}

Null

Null

Figure 10. Detector images on each guider showing the OTIS source movement for the polarity test

\section{Centroid Spiral Plot (Test Data and Ideal Sim Pattern)}

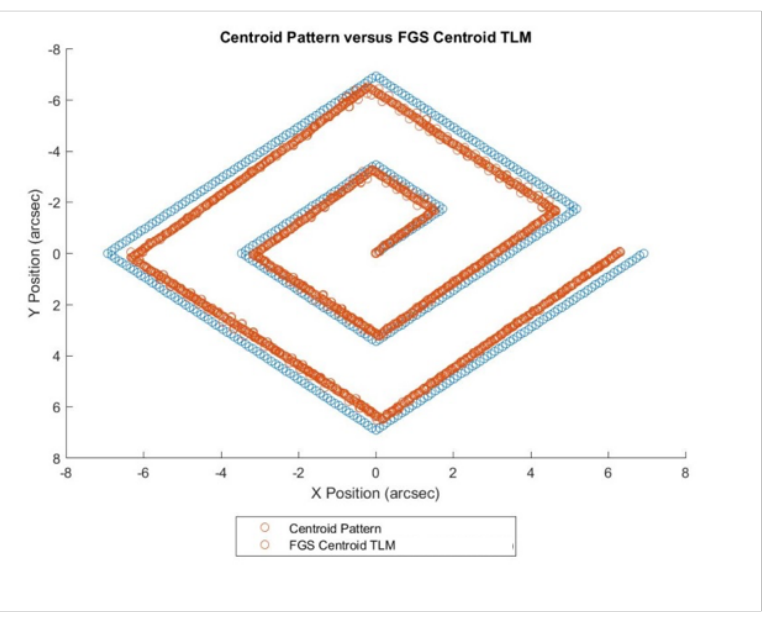

Guider 1

FSM-FGS Misalignment: $-1.3 \mathrm{deg}$

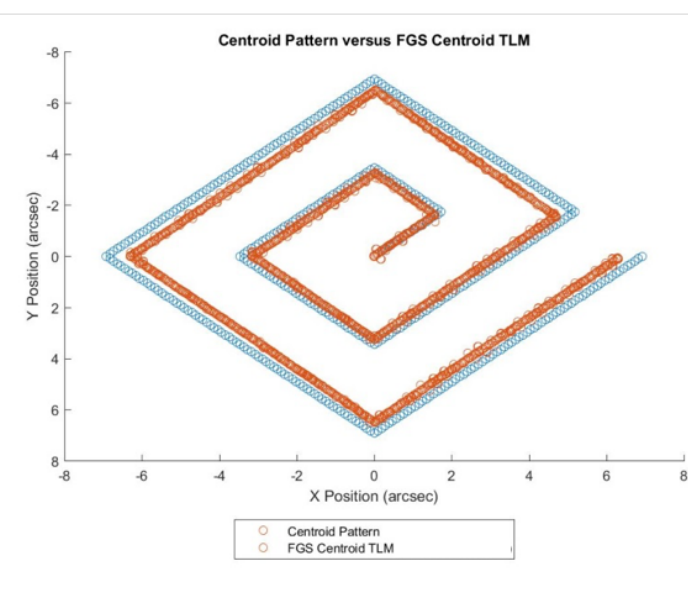

Guider 2

FSM-FGS Misalignment: 0.14 deg

Figure 21. Comparison of the commanded spiral pattern versus actual 


\subsection{Functional Test Results}

Successful closed loop was maintained during the functional test with the FGS in track mode as shown in Figure 12 for both guiders. The Figure shows the centroid positions aligned well with the commanded step pattern and also shows good agreement with predictions from simulated data.

Similarly closed loop was maintained during the functional test with FGS in fine guide mode as shown in Figure 13 for Guider 1 in both $\mathrm{X}$ and $\mathrm{Y}$ axes. When using FGS in fine guide mode the FSM motion is kept to no more than 1 guider pixel (70 mas) as the $8 \times 8$ pixel fine guide window will not be moved.

\section{Centroid Time History (Test Data and Sim Predicts)}
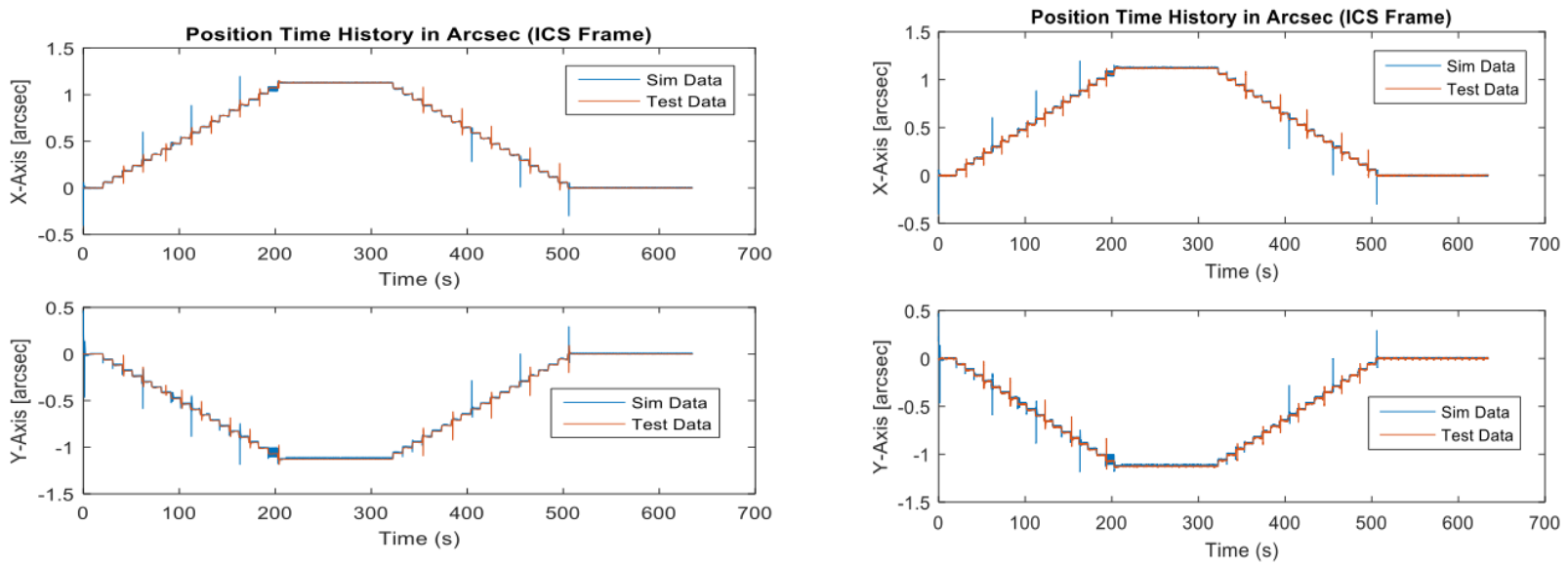

Guider 1

\section{Guider 2}

Figure 32. Closed loop centroid data for each Guider in Track mode.
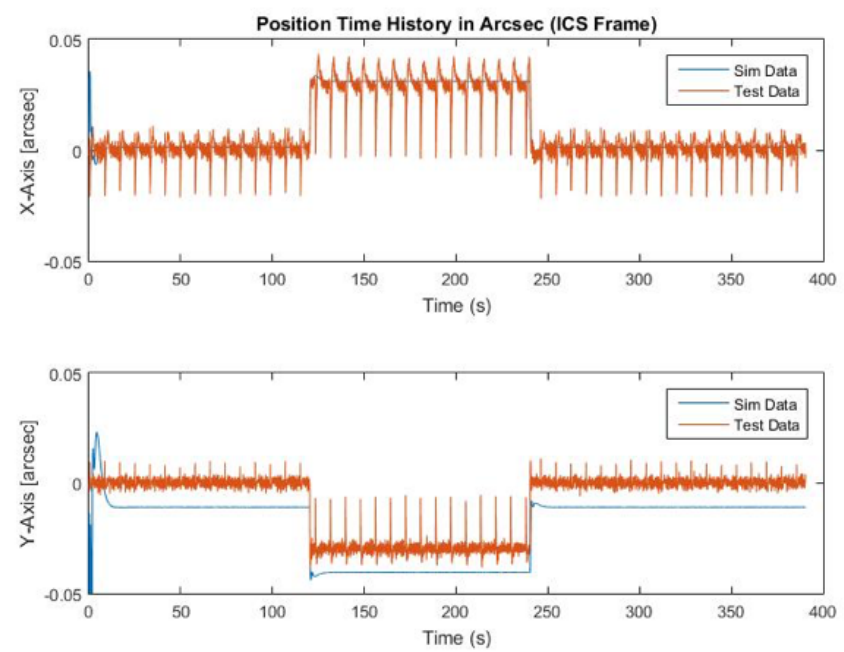

\section{Guider 1}

Figure 43. Closed loop centroid data for one Guider in Fine Guide mode. 
Closed loop Fine Guidance control was also maintained with FGS in fine guide mode with the dynamic line of sight updates commanding in $\mathrm{X}$-axis, $\mathrm{Y}$-axis and combined $\mathrm{X} / \mathrm{Y}$ axis as shown in Figure 14.

It is noticed on all the fine guide data, periodic 8 second oscillations of approximately 1 pixel. The analysis of this data confirmed that these oscillations are due to a combination of the unusual shape of the OTIS source and the detector rolling reset. The OTIS PSF shape has bright pixels in several places outside the 8x8 pixel fine guide subwindow bleeding signal into that window. A rolling reset is part of the detector read functionality, and the purpose is to clear signal regularly across the whole detector to avoid charge accumulation. When this happens the signal on the fine guide $8 \times 8$ window changes causing the centroid to move. The rolling reset clears 16 rows for each integration and 128 integrations are needed to cover the whole detector which with an integration time of $64 \mathrm{~ms}$ gives the $8 \mathrm{~s}, 0.122 \mathrm{~Hz}$ variation seen on all the closed loop fine guidance data. This phenomena will not happen on orbit as the objects to be observed will have a more uniformly centered PSF. It should also be noted that even with this oscillation, it was small enough that fine guide was never lost, and only noticed on the detailed analysis of the centroids post-test.

\section{Centroid Time History (Test Data and Sim Predicts)}

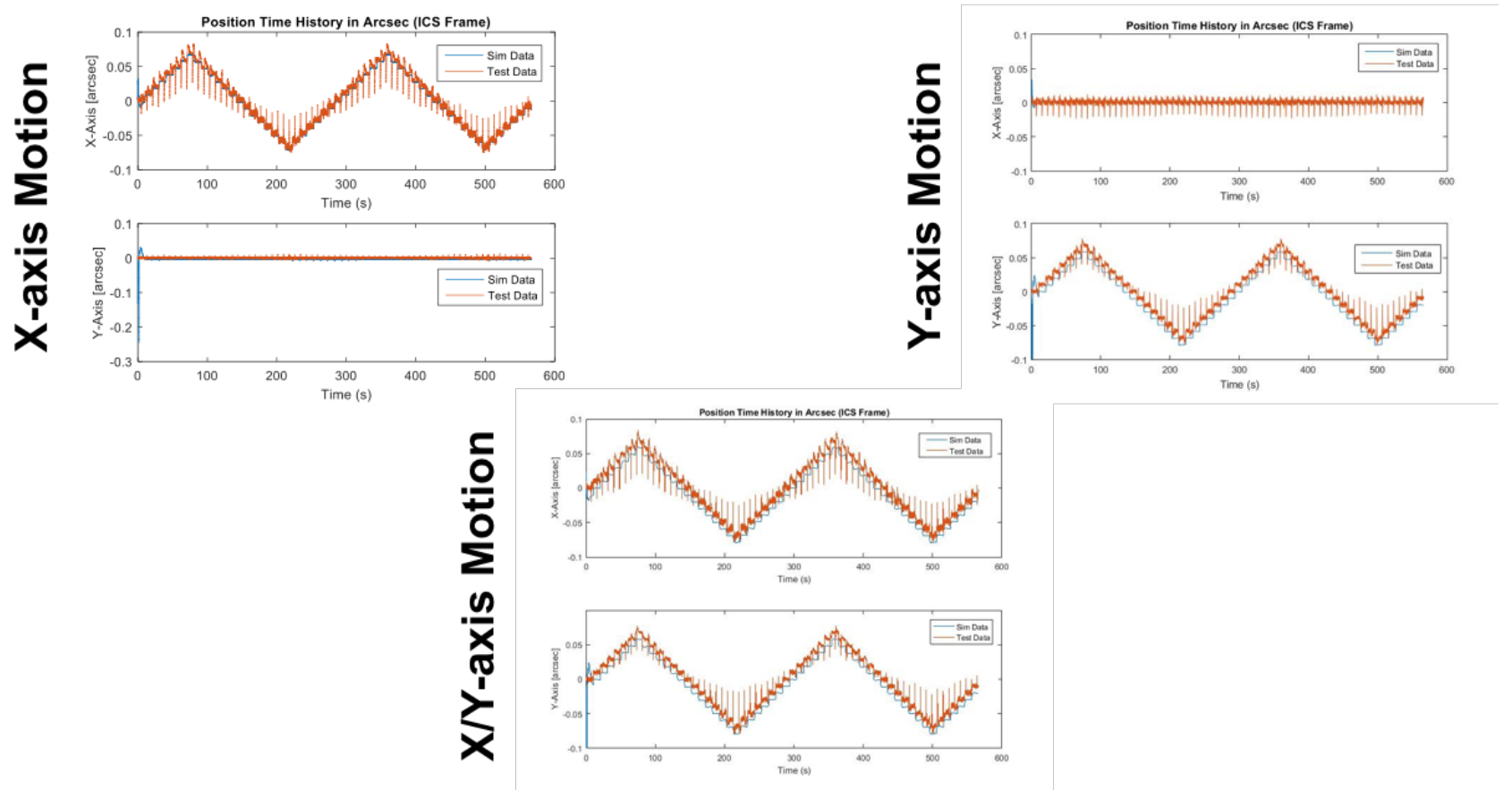

Figure 54. Closed loop centroid data for dynamic LOS motion with the Guider in fine guide mode

\subsection{Timing Performance Test Results}

The timing test showed that Fine Guidance Control was successfully maintained with FGS in fine guide mode for 10000 $\mathrm{s}$, a typical observation cadence on orbit. In parallel two other instruments (MIRI and NIRISS) were used to take images showing a typical observation scenario of guiding while taking science images.

The timing data analysis used the centroids from Guider 1 in closed loop and the calculated centroids from the 8x8 pixel images taken by Guider 2 to obtain a boundary on the $64 \mathrm{~ms}$ latency requirement. A mean value of $62.1 \mathrm{~ms}$ was obtained. Figure 15 shows centroid data for Guider 1 and the calculated centroids for Guider 2.

Bus monitors were used during the test to capture all the $64 \mathrm{~ms}$ timing packets to aid on this analysis - this included the centroid packets produced by the FGS (1), the duplicated packets copied by the Spacecraft (2) and used by the ACS (3), the command sent by the ACS to the FSM, and the reported telemetry from the FSM when its position was updated (4). 
The comparison of the packets from (1) and (2) confirmed that the duplication occurred correctly every 64 ms. The analysis of the timing from when (1) was picked up at each $64 \mathrm{~ms}$ timecode with (3) confirmed that the timing requirement was met. Information from (4) indicates that the FSM was moved in the correct amount of time to the commanded position from (3) - however that telemetry update is too slow to confirm the actual time for the FSM move which is why the image data from Guider 2 is used. Figure 16 gives an example of the complex component timing analysis.

\section{Centroid Time History (Test Data and Sim Predicts)}
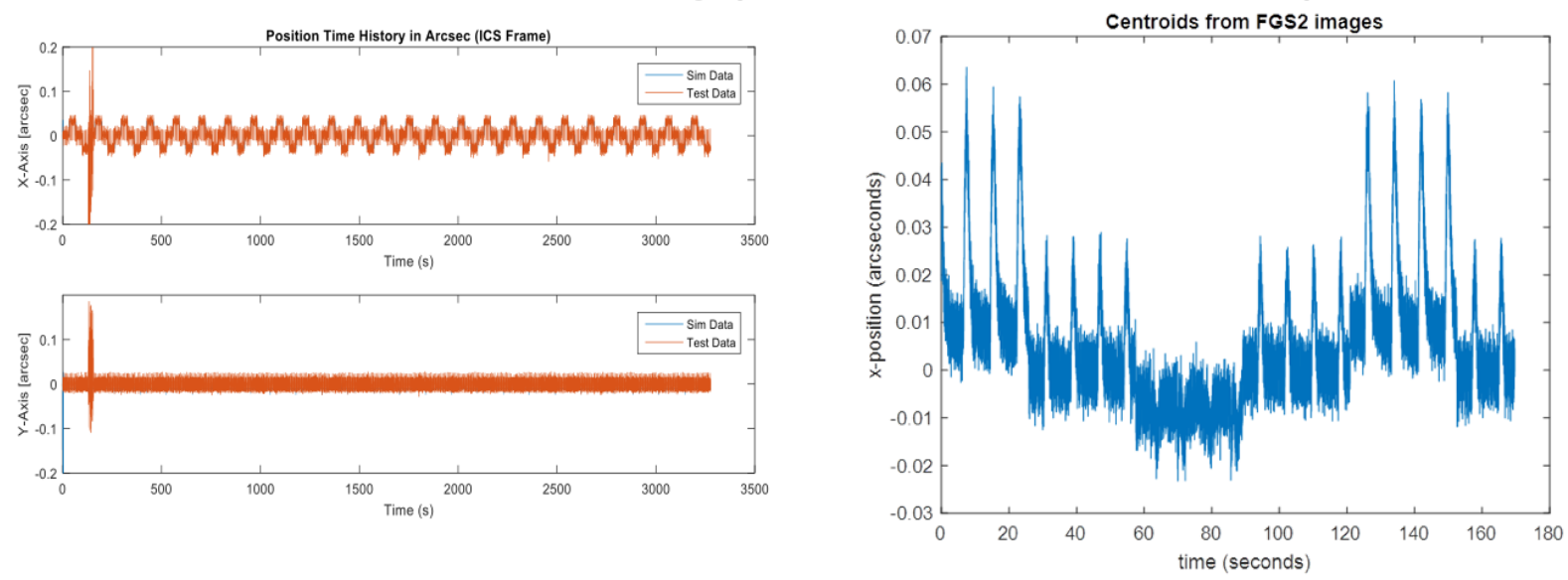

Guider 1

Guider 2

Figure 65. Closed loop centroid data for Guider 1 and Guider 2 8x8 pixel centroids

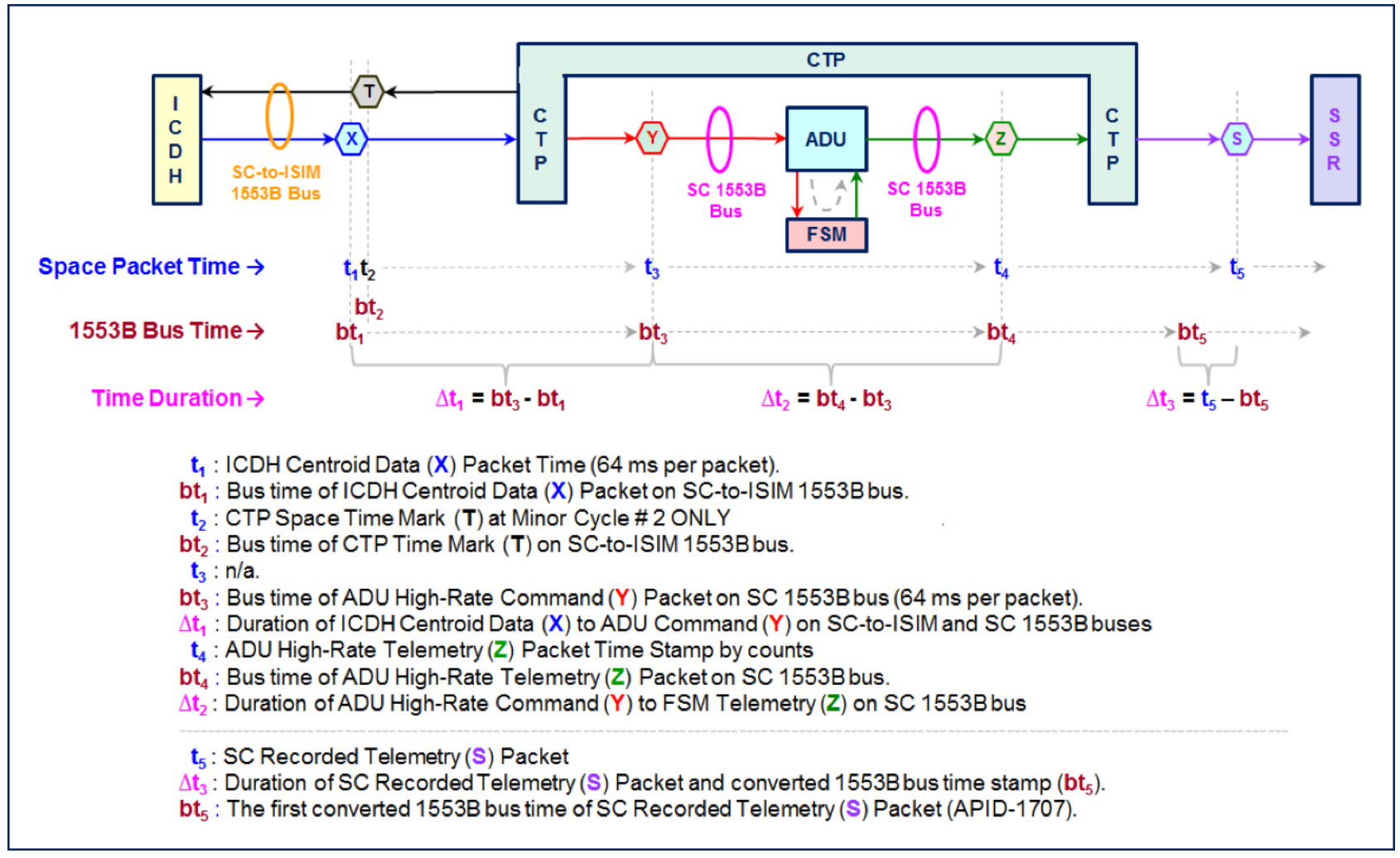

Figure 76. Example of the complicated component timing analysis 


\section{CONCLUSIONS}

Three main tests were carried out as part of the cryo testing at JSC with the flight FGS, FSM and the ACS software to validate the closed loop performance. The polarity between the FGS coordinates and the FSM axis was confirmed and the FGS tracking performance on a moving source validated. The ability to maintain closed loop and FGS lock while tracking and fine guiding with the Guide Star moving in various patterns was demonstrated as well as the timing budget. A typical observation of $10000 \mathrm{~s}$ with the Fine Guidance Controller in closed loop and another James Webb instrument taking images in parallel as it would on orbit, duplicating the data and telemetry rates that would be seen, confirmed full functionality. The successful testing identified some items to be optimized and set the Fine Guidance Controller on a good path for operations.

\section{REFERENCES}

[1] Feinberg, L. D., Clampin, M., Keski-Kuha, R., Atkinson, C., Texter, S., Bergeland, M., Gallagher, B. B."James Webb Space Telescope optical telescope element mirror development history and results," Proc. SPIE 8442, Space Telescopes and Instrumentation 2012: Optical, Infrared, and Millimeter Wave, 84422B (2012)

[2] Rieke, M., Kelly, D. and Horner, S., "Overview of James Webb Space Telescope and NIRCam's Role,” Proc. SPIE 5904, 1-8 (2005).

[3] Beichman, C. A., Rieke, M., Eisenstein, D., Greene, T. P., Krist, J., McCarthy, D., Meyer, M., and Stansberry, J., "Science opportunities with the near-IR camera (NIRCam) on the James Webb Space Telescope," Proc. SPIE 8442, 84422N (2012).

[4] Bagnasco, G. et al., "Overview of the near-infrared spectrograph (NIRSpec) instrument on-board the James Webb Space Telescope (JWST)," Proc. SPIE 6692, 66920M (2007).

[5] Birkmann, S. M. et al., “The JWST/NIRSpec instrument: update on status and performances,” Proc. SPIE 9904, in press (2016).

[6] Rieke, G. H. et al., "The Mid-Infrared Instrument for the James Webb Space Telescope, I: Introduction,” PASP Vol. 127, Issue 953, 584-594 (2015).

[7] Wright, G. S. et al., "The Mid-Infrared Instrument for the James Webb Space Telescope, II: Design and Build," PASP Vol. 127, Issue 953, 595-611 (2015).

[8] Doyon, R. et al., "The JWST Fine Guidance Sensor (FGS) and Near-Infrared Imager and Slitless Spectrograph (NIRISS)," Proc. SPIE 8442, 84422R (2012).

[9] Maszkiewicz, M., Saad, K., Rowlands, N., Doyon, R. and Hutchings, J. B., "JWST Fine Guidance Sensor and Near- Infrared Imager and Slitless Spectrograph,” Optical Payloads for Space Missions (ed S.-E. Qian), ch35, (2015).

[10] Kimble, R. A, Vila, M.B, Van Campen, J. M., et al. 'Cryo-vacuum testing of the JWST integrated science instrument module (ISIM), Proc. SPIE 9904, (2016)

[11] Rowlands, N., Beaton, A., Chayer, P., Haley, C., Midwinter, C., Volk, K., Warner, G., Zhou, J., "Updated cryogenic performance test results for the flight model JWST fine guidance sensor", Proc. SPIE 9904, Space Telescopes and Instrumentation 2016: Optical, Infrared, and Millimeter Wave, (2016)

[12] N. Rowlands, S. Delamer, C. Haley, E. Harpell, M. B. Vila, G. Warner, J. Zhou, 'Cryogenic performance test results for the flight model JWST fine guidance sensor'. SPIE, 8442-130, (2012)

[13] Vila, M. B., Rowlands, N., Desaulniers, D-L., Evans., C. 'JWST Fine Guidance Sensor Performance Analysis'. CASI ASTRO 2008, Proceedings, 62, (2008)

[14] Meza, L., Tung, F., Anandakrishnan, S., Spector, V., Hyde, T.'Line of Sight Stabilization of James Webb Space Telescope', $27^{\text {th }}$ Annual AAS Guidance and Control Conference, AAS 05-002, (2005) 


\section{ACKNOWLEDGMENTS}

The authors gratefully acknowledge all the dedicated members that were part of planning and running this test procedure at the OTIS cryo vacuum testing. In particular the group at Northrop Grumman which was tasked with designing this test, the group at Honeywell who are the instrument development team, the Optics group on the JWST project at Goddard Space Flight Center that contributed to the analysis, and the GSFC team that contributed to the test planning, validation and analysis. 